\title{
Influence of Surface Oxygen Groups on V(II) Oxidation Reaction Kinetics
}

\author{
Susan M.Taylor ${ }^{a}$, Alexandra Pătru ${ }^{a}$, EmilianaFabbri $^{a}$, Thomas J. Schmidt ${ }^{a, b}$ \\ ${ }^{\text {a} E l e c t r o c h e m i s t r y ~ L a b o r a t o r y, ~ P a u l ~ S c h e r r e r I n s t i t u t, ~} 5232$ Villigen PSI, Switzerland \\ baboratory of Physical Chemistry, ETH Zurich, 8093 Zurich, Switzerland
}

\begin{abstract}
The role of surface oxygen groups on the kinetics of the V(II) oxidation reaction was studied on modified glassy carbon (GC) electrodes by cyclic voltammetry (CV) and electrochemical impedance spectroscopy (EIS). The reaction was found to be sensitive to the presence of oxygen groups on the electrode surface. Higher $\mathrm{O} / \mathrm{C}$ ratios determined by $\mathrm{X}$-ray photoelectron spectroscopy (XPS) corresponded to higher reactivities and lower charge transfer resistances measured in a $1 \mathrm{M}$ V(II) electrolyte. The stability of an oxidised GC surface was also investigated in a $1 \mathrm{M}$ V(II) electrolyteby potential holding and cyclingexperiments. It was found that after holding and cycling to successively more negative potentials up to $-0.8 \mathrm{~V} / \mathrm{RHE}$, the electrode surface lost its initial reactivity. Keywords: Vanadium (II) oxidation; Vanadium flow battery; Glassy carbon; stability; reaction kinetics
\end{abstract}

*Corresponding author: Tel +41563102318. Email: alexandra.patru@psi.ch (Alexandra Pătru) 


\section{Introduction}

The surface chemistry and structure of the carbon electrodes used in the all-vanadium redox flow cell have been found to play a key role in improving the kinetics of both the V(II) oxidation and $V(V)$ reduction reactions[1-4]. Significant efforts have been made to understand and optimise the surface properties of the carbon electrodes and identify the limiting half-cell reaction in thissystem[5, 6]. A common approach to improving electrode performance has been the introduction of functional groups, particularly oxygen-containing groups, onto the electrode surface. The observed improvements in electrode performance have been attributed to a wetting or surface area effect and/or a catalytic effect of these surface groups[1, 7, 8]. However, the exact role of surface oxygenated groupson the kinetics of both reactions is unclear and the limiting half-cell reaction is still a topic of debate. Contrary to reports that $V(V)$ reduction has the slower reaction kinetics of the two redox reactions, recent studies have shownthat the V(II) oxidation limits the overall cell performance[5, 7-15].It has also been suggested that surface oxides could play a catalytic role in improving the kinetics of this reaction[8-10, 15].Early studies by McDermott et al. showed that V(II) oxidation follows an inner-sphere reaction mechanism involving bridging of hydrated V(II) ions with the surface oxide groups[12]. While the catalytic role of surface oxides for V(II) oxidation in the all-vanadium system has been suggested by several authors, discrepancies still remain in the literature regarding this point. It is the purpose of this study to gain further insight into the influence of surface oxygen groups on the kinetics of the V(II) oxidation reaction.Glassy carbon electrodes treated by oxidative and surface roughening methods were used to study V(II) oxidation reaction kinetics by cyclic voltammetry (CV) and electrochemical impedance spectroscopy (EIS). Trends of reactivityvs. oxygen content determined by X-ray photoelectron spectroscopy (XPS)were investigated. Finally, the stability of surface oxygen groups in the potential range of V(II/III) oxidation/reduction was studied. 


\section{Experimental}

\subsection{Electrolyte preparation}

A 1 M V(II) electrolyte solution was prepared by electrolysis of 1 M V(IV) solution $\left(\mathrm{VOSO}_{4} \cdot \mathrm{nH}_{2} \mathrm{O}\right.$, Alfa Aesar 99.9\%, dissolved in $2 \mathrm{M} \mathrm{H}_{2} \mathrm{SO}_{4}$, Sigma Aldrich 99.999\%) using a commercial redox flow cell system(Scribner ${ }^{\circledR}$ ) and carbon paper electrodes (SGL39AA). The concentration of $\mathrm{V}(\mathrm{II})$ was confirmed by UV-visible spectroscopy (Metrohm ${ }^{\circledR}$ ) to be $1 \mathrm{M}$.

\subsection{Electrode materials and treatment methods}

Glassy carbon (GC) disk electrodes were suppliedbyHochtemperaturwerkstoffe GmbH (Sigradur ${ }^{\circledR} \mathrm{G}, 5 \mathrm{~mm}$ OD x $4 \mathrm{~mm}$ thickness, mirror polished). The GC electrodes were treated oxidatively, byacid and electrochemical treatmentsand non-oxidatively by abrasive polishing on sandpaper (3M ${ }^{\mathrm{TM}}$ Grit size-600). A mixed treatment involving abrasive polishing followed by electrochemical oxidation was also carried out (see [6]for detailed experimental conditions).Five GC electrode surfaces were investigated in this studyusing the same nomenclature as in our previous study on $\mathrm{V}(\mathrm{V})$ reduction[6]:pristine (GC-P), acid treated (GC-A), electrochemically treated (GC-EC), rough (GC-R) and mixed treatment (GC-R+EC).

\subsection{Surface characterisation}

X-ray photoelectron spectroscopy (XPS) was used to determine the oxygen content (O/C ratio) of the treated GC electrodes. A VG ESCALAB 220iXL spectrometer (Thermo FischerScientific) equipped with an Al Ka monochromatic source and amagnetic lens system was used. High resolution spectra of the C1s and O1s peaks were used to determine the surface oxygen to carbon ratio (O/C ratio) for all electrode samples.All curves were fitted according to a Gaussian-Lorentzian function.Background subtraction was performed according to the Shirley method, and the atomic sensitivity factors (ASF) of Scofield were applied to estimate the atomic composition.

\subsection{Electrochemical characterisation}

Electrochemical measurements were carried out using a standard three-electrode setup with a platinum-mesh counter electrode and a $\mathrm{Hg} / \mathrm{Hg}_{2} \mathrm{SO}_{4}$ reference electrode(Gamry instruments Inc. ${ }^{\circledR}$ ) at room temperature. Cyclic voltammograms (CVs) were recorded at $50 \mathrm{mV} / \mathrm{s}$ in an argon 
purged $1 \mathrm{M}$ V(II) electrolyte and reported against the reversible hydrogen electrode (RHE). All CVs were correctedduring data analysis for ohmic resistance. Electrochemical impedance spectroscopy (EIS) measurements were carried out at $\sim 10 \mathrm{mV}$ overpotential for V(II) oxidation with a voltage amplitude of $10 \mathrm{mV}$ in the frequency range: $1 \mathrm{MHz}-50 \mathrm{mHz}$. The experimental data were fitted to the equivalent electrical circuit by a randomize-simplex procedure using Z Fit EC Lab ${ }^{\circledR}$ software.In order to assess the stability of an oxidised GC electrode in the potential range of the $\mathrm{V}(\mathrm{II}) / \mathrm{V}(\mathrm{II})$ redox couple, potential holding and cyclingexperiments were carried out in $1 \mathrm{M} \mathrm{V(II)} \mathrm{electrolyte.} \mathrm{For} \mathrm{potential} \mathrm{holding} \mathrm{measurements,} \mathrm{the} \mathrm{electrode} \mathrm{was} \mathrm{held} \mathrm{at} \mathrm{OCV} \mathrm{for}$ 10 seconds before recording the initial $\mathrm{CV}$, then three negative potentials were applied successively to the electrode: $-0.6,-0.7,-0.8 \mathrm{~V} / \mathrm{RHE}$ for 10 seconds each. A CV was recorded after each potential holding step. Cycling experiments were carried out in two potential ranges: potential range $1(\Delta \mathrm{E} 1)$, from -0.6 to $0.2 \mathrm{~V} / \mathrm{RHE}$ and potential range $2(\Delta \mathrm{E} 2)$, from -0.8 to 0.2 V/RHE. 150 cycles were recorded for each potential range. The surface oxygen content (O/C) was determined by XPS after the final potential holding step of $-0.8 \mathrm{~V} / \mathrm{RHE}$ and after cycling in $\Delta \mathrm{E} 2$.

\section{Results and Discussion}

\subsection{Effect of electrode treatment on V(II) oxidation reaction kinetics}

The V(II) oxidation reaction:

$V^{2+} \rightleftharpoons V^{3+}+e^{-}$

was studied at the modified GC electrodes to investigate the influence of surface oxygen groups on electrode reactivity. The peak potential separation $\left(\Delta \mathrm{E}_{\mathrm{p}}\right)$ measured by CVand the charge transfer resistance $\left(\mathrm{R}_{\mathrm{CT}}\right)$ measured by EIS were determinedto give an indication of electrode reactivity[16].Fig. 1 shows the CVs and Nyquist impedance plotsof the pristine and modifiedGC electrodes measured in V(II) solution. The reactivity data are summarised in Table 1. 

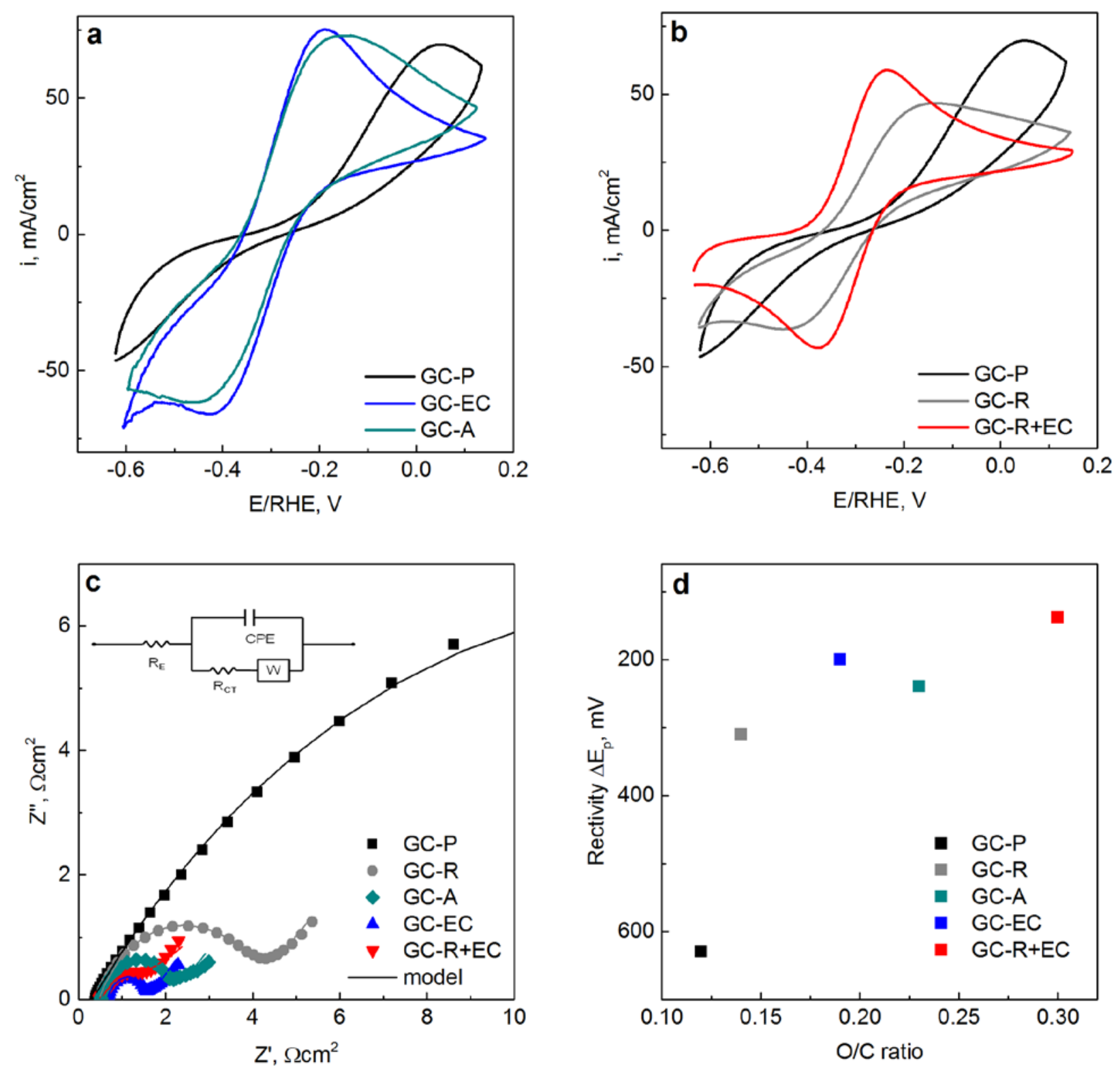

Fig. 1.Cyclic voltammograms measured at $50 \mathrm{mV} / \mathrm{s}$ in $1 \mathrm{M} \mathrm{V(II)/2} \mathrm{M} \mathrm{H}_{2} \mathrm{SO}_{4}$ for GC electrodes subject to (a) oxidative treatments and (b) abrasive polishing and mixed treatment (c)Nyquist impedance plots for all GC electrodes, markers show experimental data, solid lines show the fitted data using the equivalent electrical circuit diagram shown in the inset (d) reactivity $\left(\Delta E_{p}\right)$ versus $\mathrm{O} / \mathrm{C}$ ratio determined by XPS for all GC electrodes. 
Table 1. Summary ofCV, XPS and EIS data

\begin{tabular}{|lllll|}
\hline Electrode & $\mathbf{O} / \mathbf{C}$ ratio & Reactivity & $\mathbf{R}_{\mathbf{C T}}$ & $\chi^{2}$ \\
& & $\Delta \mathbf{E}_{\mathbf{p}}$ & $\mathbf{\Omega .}^{2}$ & \\
& & $\mathbf{m V}$ & & \\
GC- & 0.30 & 140 & & 0.04 \\
R+EC & & & 0.6 & \\
GC-EC & 0.19 & 200 & 0.9 & 0.02 \\
GC-A & 0.23 & 240 & 1.7 & 0.02 \\
GC-R & 0.14 & 310 & 3.7 & $9.8 \mathrm{E}-3$ \\
GC-P & 0.12 & 630 & 28 & 0.03 \\
\hline
\end{tabular}

Where: $\boldsymbol{R}_{C T}-$ charge transfer

resistance, $\chi^{2}-$ fit accuracy

The GC-EC, GC-A and GC-R+EC electrodes showed the best reactivity towards V(II) oxidation. These surfaces showed the smallest $\Delta \mathrm{E}_{\mathrm{p}}$ values in Fig. 1a and $1 \mathrm{~b}$ andanalysis of the impedance data in Fig. 1c revealed a decrease in the charge transfer resistance. Thisindicates an enhancement of the reaction kinetics of V(II) oxidation at these three surfaces.XPS analysis confirmed an increase in the oxygen content (O/C ratio) after treatment for these three surfaces (see Table 1).It is notable that the roughened GC electrodewith a low $\mathrm{O} / \mathrm{C}$ ratio,showed a poor reactivity towards V(II) oxidation. Thesmallimprovement in peak separation for GC-R compared to GC-P could be attributed to either a kinetic and/or a diffusional effect[17]. After electrochemical oxidationof the GC-R and a consequent increase in O/C ratio, asubstantial improvement in reactivity was observed at the GC-R+EC surface. The effect of defect exposure by roughening the GC surface did not have the same positive effect on reactivity as was seen for the $\mathrm{V}(\mathrm{V})$ reduction reaction in our previous study [6]. Only after the introduction of surface oxygen groups was a notable reactivity improvement observed. A plot of oxygen content $(\mathrm{O} / \mathrm{C}$ ratio) versus peak separation $\left(\Delta \mathrm{E}_{\mathrm{p}}\right)$ in Fig. 1d indicates that the $\mathrm{V}(\mathrm{II})$ oxidation reaction is sensitive to the amount of oxygen present on the surface. Higher $\mathrm{O} / \mathrm{C}$ ratios correspond to better reactivity and low $\mathrm{R}_{\mathrm{CT}}$. The observations made here are in line with several earlier and more recent studies involving V(II) oxidation at oxidised carbon surfaces [8], [9], [12], [18]. It was 
suggested previously that $\mathrm{V}$ (II) oxidation follows an inner-sphere reaction mechanism involving surface oxygen groups[18]. Thus unlikeV(V) reduction, V(II) oxidation appears to be sensitive to the presence of surfaceoxygen groups on the carbon electrode.

\subsection{Stability of surface oxygen groups}

The V(II)/V(III) redox couple occurs at a standard redox potential of $-0.26 \mathrm{~V} / \mathrm{SHE}$. This is well below 0V/SHE where it is common for an oxidised surface to undergo reduction processes[18]. We have seen in section 3.1 thatthe V(II) oxidation reaction is sensitive to the surface oxygen content. Thus the question of stability of an oxidised carbon surface in this potential range becomes crucial if oxidised electrodes are to be used in an applied system. The stability of an electrochemically oxidised glassy carbon surface was studied by potential holding and cycling experimentsand the effect on peak separation $\left(\Delta \mathrm{E}_{\mathrm{p}}\right)$ was studied (Fig. 2).
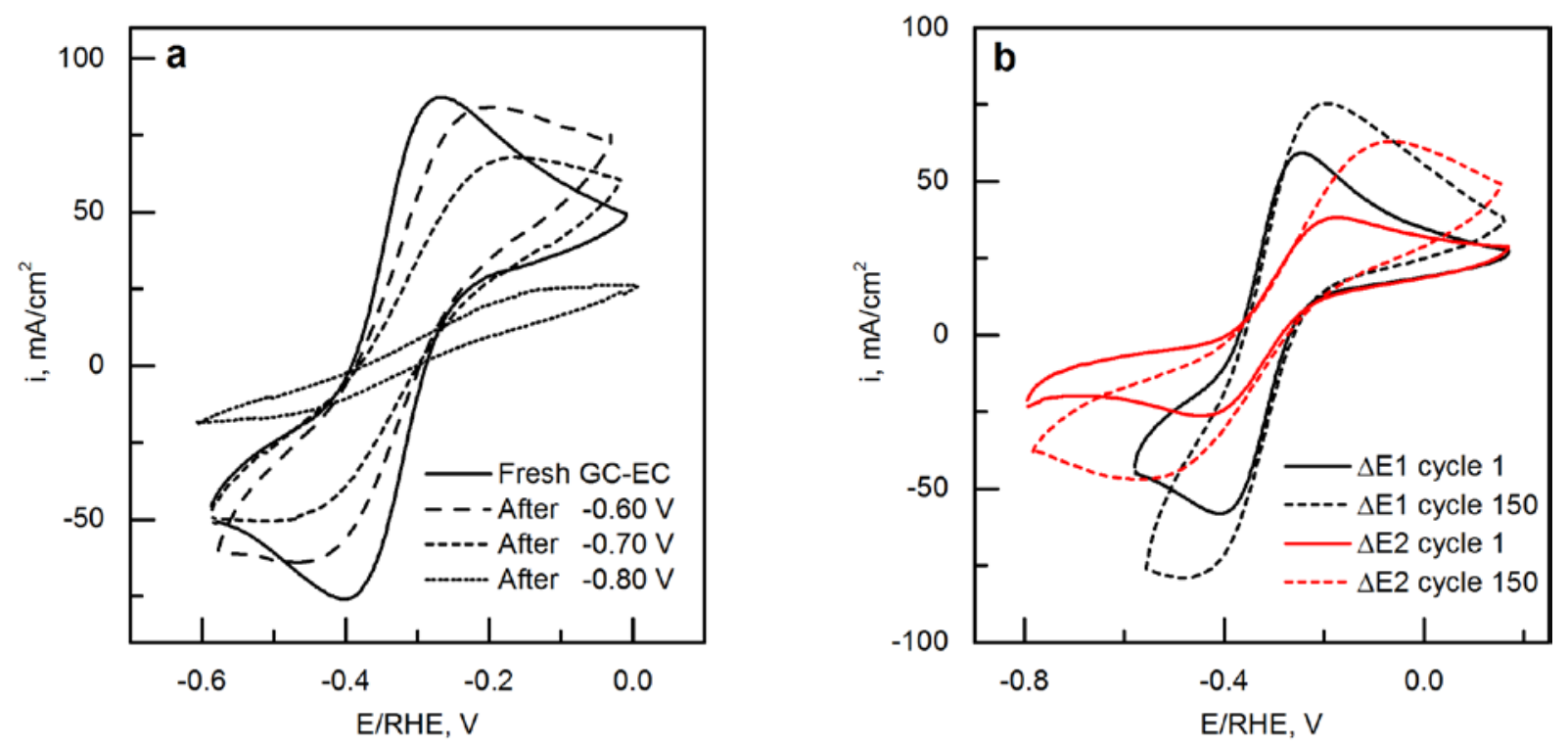

Fig. 2 - Cyclic voltammograms measured at $50 \mathrm{mV} / \mathrm{s}$ in $1 \mathrm{M} \mathrm{V(II)/2} \mathrm{M} \mathrm{H}_{2} \mathrm{SO}_{4}$ for a GCECelectrode (a) subject to potential holding steps at OCV, -0.6, $-\mathbf{0 . 7}$ and $\mathbf{- 0 . 8} \mathrm{V} / \mathrm{RHE}$ for 10 seconds per step and (b) 150 cycles in two different potential ranges $\Delta \mathrm{E} 1$ and $\Delta E 2$, CVs show the $1^{\text {st }}$ and $150^{\text {th }}$ cycle.

The CVs in Fig. 2a show that after the first potential holding step at -0.6V/RHE, the peak separation has increased, indicating a loss in reactivity at the GC-EC surface. After progressive potentiostatic steps at $-0.7 \mathrm{~V} / \mathrm{RHE}$ and finally $-0.8 \mathrm{~V} / \mathrm{RHE}$, the peak separation grows larger and the surface loses its initial reactivity. This is in line with recent findings by Bourke et al. and 
Miller et al.[8, 15].Similarly, after cycling the GC-EC electrode in two different potential ranges, the electrode surface loses its initial reactivity. A larger peak separation is observed after 150 cycles in $\Delta \mathrm{E} 2$ than $\Delta \mathrm{E} 1$. Both these experiments highlight the detrimental effects of progressively more negativepotentials on electrode reactivity. The $\mathrm{O} / \mathrm{C}$ ratio decreased from 0.19 to 0.14 after potential holding at $-0.8 \mathrm{~V} / \mathrm{RHE}$ and to 0.17 after cycling in $\Delta \mathrm{E} 2$. The decrease in $\mathrm{O} / \mathrm{C}$ ratio in both cases does not correspond directly with the decrease in reactivity. This is an indication that other surface phenomena such as passivation may also contribute to the loss in reactivity,as has been suggested previously [19]. Furthermore the type of carbon may also play a role in the observed stability of an oxidised carbon electrode. The same experiments carried out on an oxidised edge plane pyrolytic graphite electrode revealed more stable behaviour than the EC-GC under the same stability test conditions. Furtherstudies on the stability of oxidised carbon surfaces is needed to better understand the nature of the potential degradation observed here. Nonetheless it is evident that the negative potential limit has a detrimental effect on the stability of an oxidised carbon surface. This finding is particularly relevant for applied systemsin which the electrode at the V(II) side is exposed to negative potentials for extended periods of time during charging.

\section{Conclusions}

In this study we have explicitly shownthat improvements in the V(II) oxidation reaction kinetics observed by EIS and CV correspond to an increase in the total surface oxygen content (O/C).Furthermore, the stability of surface oxygen groups in the potential range of V(II) oxidation appears to be a critical issue. An oxidised GC surface loses its initial reactivity after a series of potentiostatic steps and cycling measurements. The negative potential limit seems to have a detrimental effect on the stability of an oxidised carbon surface and further investigations into the mechanisms behind this effect are required.Oxygen treatment may not be the most effective long-term solution to improving V(II) oxidation kinetics at the negative electrodein an applied system. The stability could be a decisive issue for long-term operation andalternative, stablesurface treatments and materials should be explored. Nonetheless, there is still much to be learned regarding the mechanism of V(II) oxidation and the catalytic role of surface oxygen groups. Further understanding of the reaction mechanism will help in optimising and developing a more suitable and stablenegative electrode material for this reaction. 


\section{Acknowledgements}

The authors thank The Swiss National Science Foundation for their financial support within the REPCOOL project (Grant No. 147 661).

\section{References}

[1] J. Maruyama, T. Hasegawa, S. Iwasaki, T. Fukuhara, and M. Nogami, “Mechanism of dioxovanadium ion reduction on oxygen-enriched carbon surface,” J. Electrochem. Soc., vol. 160, no. 8, pp. A1293-A1298, Jun. 2013.

[2] J. Melke, P. Jakes, J. Langner, L. Riekehr, U. Kunz, Z. Zhao-Karger, A. Nefedov, H. Sezen, C. Wöll, H. Ehrenberg, and C. Roth, "Carbon materials for the positive electrode in all-vanadium redox flow batteries,” Carbon N. Y., vol. 78, pp. 220-230, Nov. 2014.

[3] J. Friedl, C. M. Bauer, A. Rinaldi, and U. Stimming, "Electron transfer kinetics of the VO2+/VO2+ - Reaction on multi-walled carbon nanotubes," Carbon N. Y., vol. 63, pp. 228-239, Nov. 2013.

[4] L. Cao, M. Skyllas-Kazacos, and D.-W. Wang, "Effects of surface pretreatment of glassy carbon on the electrochemical behavior of $\mathrm{V}(\mathrm{IV}) / \mathrm{V}(\mathrm{V})$ redox reaction,” J. Electrochem. Soc., vol. 163, no. 7, pp. A1164-A1174, 2016.

[5] E. Agar, C. R. Dennison, K. W. Knehr, and E. C. Kumbur, "Identification of performance limiting electrode using asymmetric cell configuration in vanadium redox flow batteries," J. Power Sources, vol. 225, pp. 89-94, 2013.

[6] S. M. Taylor, A. Patru, D. Streich, M. El Kazzi, E. Fabbri, and T. J. Schmidt, "Vanadium (V) reduction reaction on modified glassy carbon electrodes - Role of oxygen functionalities and microstructure,” Carbon N. Y., vol. 109, pp. 472-478, 2016.

[7] A. M. Pezeshki, J. T. Clement, G. M. Veith, T. A. Zawodzinski, and M. M. Mench, "High performance electrodes in vanadium redox flow batteries through oxygen-enriched thermal activation,” J. Power Sources, vol. 294, pp. 333-338, 2015.

[8] A. Bourke, M. A. Miller, R. P. Lynch, X. Gao, J. Landon, J. S. Wainright, R. F. Savinell, and D. N. Buckley, "Electrode kinetics of vanadium flow batteries: contrasting responses of $\mathrm{V}^{\mathrm{II}}-\mathrm{V}^{\mathrm{III}}$ and $\mathrm{V}^{\mathrm{IV}}-\mathrm{V}^{\mathrm{V}}$ to electrochemical pretreatment of carbon,” J. Electrochem. Soc., vol. 163, no. 1, pp. A5097-A5105, 2016.

[9] H. Fink, J. Friedl, and U. Stimming, "The faster half-cell in a vanadium redox flow battery is determined by the electrode composition,” J. Phys. Chem. C, vol. 120, no. 29, pp. 15893-15901, 2016.

[10] D. Dixon, D. J. Babu, J. Langner, M. Bruns, L. Pfaffmann, A. Bhaskar, J. J. Schneider, F. Scheiba, and H. Ehrenberg, "Effect of oxygen plasma treatment on the electrochemical performance of the rayon and polyacrylonitrile based carbon felt for the vanadium redox flow battery application,” J. Power Sources, vol. 332, pp. 240-248, 2016.

[11] C.-N. Sun, F. M. Delnick, D. S. Aaron, A. B. Papandrew, M. M. Mench, and T. A. Zawodzinski, "Resolving losses at the negative electrode in all-vanadium redox flow 
batteries using electrochemical impedance spectroscopy,” J. Electrochem. Soc., vol. 161, no. 6, pp. A981-A988, 2014.

[12] C. A. McDermott, K. R. Kneten, and R. L. McCreery, "Electron transfer kinetics of aquated $\mathrm{Fe}+3 /+2, \mathrm{Eu}+3 /+2$, and $\mathrm{V}+3 /+2$ at carbon electrodes inner sphere catalysis by surface oxides,” J. Electrochem. Soc., vol. 140, no. 9, pp. 2593-2599, 1993.

[13] D. Aaron, C.-N. Sun, M. Bright, A. B. Papandrew, M. M. Mench, and T. A. Zawodzinski, "In situ kinetics studies in all-vanadium redox flow batteries," ECS Electrochem. Lett., vol. 2, no. 3, pp. A29 - A31, 2013.

[14] J. Langner, M. Bruns, D. Dixon, A. Nefedov, C. Wöll, F. Scheiba, H. Ehrenberg, C. Roth, and J. Melke, "Surface properties and graphitization of polyacrylonitrile based fiber electrodes affecting the negative half-cell reaction in vanadium redox flow batteries," $J$. Power Sources, vol. 321, pp. 210-218, 2016.

[15] M. A. Miller, A. Bourke, N. Quill, J. S. Wainright, R. P. Lynch, D. N. Buckley, and R. F. Savinell, "Kinetic study of electrochemical treatment of carbon fiber microelectrodes leading to in situ enhancement of vanadium flow battery efficiency," J. Electrochem. Soc., vol. 163, no. 9, pp. A2095-A2102, 2016.

[16] A. J. Bard and L. R. Faulkner, Electrochemical Methods: Fundamentals and Applications, Second edition. Hoboken NJ, 2001: John Wiley \& Sons.

[17] E. O. Barnes, X. Chen, P. Li, and R. G. Compton, "Voltammetry at porous electrodes : a theoretical study,” J. Electroanal. Chem., vol. 720-721, pp. 92-100, 2014.

[18] R. L. McCreery, K. K. Cline, C. A. McDermott, and M. T. McDermott, "Control of reactivity at carbon electrode surfaces,” Colloids Surfaces A Physicochem. Eng. Asp., vol. 93, no. C, pp. 211-219, 1994.

[19] S. Rudolph, U. Schröder, and I. M. Bayanov, "On-line controlled state of charge rebalancing in vanadium redox flow battery,” J. Electroanal. Chem., vol. 703, pp. 29-37, 2013. 